\title{
NEW APPROACH FOR ANALYSIS OF THE X-RAY DATA IN CORES OF GALAXY CLUSTERS: BINNING ON THE CONCENTRATION RINGS, CONTOUR BINNING, AND WAVELET TRANSFORMS
}

\author{
I. B. Vavilova \& Iu. V. Babyk \\ Main Astronomical Observatory of the National Academy of Sciences of Ukraine, \\ 27 Zabolotnogo str., Kyiv 03143, Ukraine, irivav@mao.kiev.ua
}

ABSTRACT. We discuss mathematical methods for analysis of the X-ray observational data to obtain a distribution of the main physical parameters of $\mathrm{X}$-ray galaxy clusters. First of all, this is the binning of galaxy cluster imagery on the concentration rings around the central parts. It allows us to calculate the integrated amount of pixels, which contain the information on temperature and other parameters of hot diffuse gas, cooling flows etc., as well as to detach the central engine of galaxy cluster. The another approach is the so-called "contour binning algorithm", which allows us to select contours on an adaptively smoothed map in such a manner that the generated bins closely fit the surface brightness. This method is mostly informative when the spectral properties follow surface brightness and/or their distribution is not smooth. We compare these methods using the Chandra observational data for A2029, A2107, and A2151 galaxy clusters. We consider a wavelet analysis likely the "Mexican Hat" as an additional and important approach for the X-ray image processing to determine not only the regions of cores in clusters but also substructures occupied by galaxies and ICM regions while processing their X-ray image contour binning. We confirm that the detachment of substructures should precede further research because of the existence of subclustering imposes doubt in the validity of the hydrostatic eqilibrium hypothesis of the X-ray gas when the dynamical matter distribution and the profiles of various physical parameters along radii are considered. A combined application of these three techniques can significantly complement our understanding of the physical processes that occur in $\mathrm{X}$-ray galaxy clusters.

Key words: X-ray astronomy, contour binning algorithm, wavelet analysis - galaxy clusters: individual: A2029, A2107, A2151
АБСТРАКТ. Ми обговорюємо математичні методи аналізу спостережних даних для одержання розподілу основних фізичних параметрів рентгенівських скупчень галактик. Перш за все, це розбиття зображення скупчення галактик на концентричні кільця навколо його центру. Це дозволяє розрахувати інтегровану кількість пікселів, що містять інформацію про температуру та інші параметри гарячого дифузного газу, потоки охолодження тощо, а також виокремити центральну, найяскравішу частину скупчення галактик. Інший підхід - це так званий "алгоритм контурного розбиття", який дозволяє окреслити контури на адаптивно згладженому зображенні у такий спосіб, що згенеровані області відповідають поверхневій яскравості всього зображення скупчення, i $€$ найбільш інформативним, якщо спектральні властивості узгоджуються з розподілом поверхневої яскравості i/або цей розподіл не $є$ згладженим. Ми порівнюємо ці методи 3 використанням даних космічної обсерваторії "Чандра" для скупчень галактик А2029, А2107 та A2151. Як додатковий i важливий підхід обробки зображень ми розглядаємо застосування хвилькового аналізу, типу перетворення "Мексиканський капелюх", щоб визначити не тільки центральну частину скупчення галактик, але й підструктури, зайняті галактиками або областями міжгалактичного середовища, під час обробки їхнього контуру рентгенівського зображення. Ми підтверджуємо, що виокремлення підструктур має передувати подальшому дослідженню, оскільки іхня присутність не дозволяє застосовувати гіпотезу 
гідростатичної рівноваги рентгенівського газу при розгляді розподілу динамічної матерії та профілів різних фізичних параметрів вздовж радіусу скупчення галактик. Комбіноване застосування цих трьох методик може суттєво доповнити наше розуміння фізичних процесів, що відбуваються в $\mathrm{X}$-скупченнях галактик.

Ключові слова: рентгенівська астрономія, алгоритм контурного розбиття, хвильковий аналіз - скупчення галактик: А2029, А2107, А2151.

\section{Introduction}

The success of high-energy astrophysics is rapid and mostly connected with space-born X-ray and gammaray telescopes. For example, the first space-born fully imaging X-ray telescope Einstein was launched by the NASA in 1978. This observatory with an angular resolution of 3-5 arcsec has resolved over $7000 \mathrm{X}$ ray sources. Over the next 20 years, with the launch of X-ray observatories of the third generation likely Chandra and XMM-Newton, the sensitivity of Xray instruments have been increasing over 10 billion times. This increasing will continue with forth generation of space telescopes such as XARM (Hitomi-II; Toshiro et al., 2018).

In the X-ray astronomy the amount of light available to compose an image is limited. This leads to what you need to assume image processing routines for translating X-ray light, which is beyond human vision, into imagery in a scientifically accurate way. There is no an immediate answer of describing how the observatory are able to see the $\mathrm{X}$-ray photons. The most care in $\mathrm{X}$ ray astronomy is taken to portray $\mathrm{X}$-ray objects truthfully. We note that our sky is not bright in X-ray light. Thus, the X-ray observations with exposure time of 5000 seconds might provide less than a 1000 individual $\mathrm{X}$-ray photons for a celestial object in dependence on its intensity. X-ray detectors are the photon-counting instruments and the basic X-ray data usually comprise lists of events and object's properties. The basic data file includes information about the time-tagged events, each with a position (in detector and celestial coordinates) and an energy. Thus, each event can be thought of as occupying a position in a $4 \mathrm{D}$ space. The event may have other attributes of interest, for example, the pattern of pixels on CCDs from which the charge for this event was accumulated. It is often possible to increase signal-to-noise by selecting on these secondary attributes. After filtering the events as required we project them onto $1 \mathrm{D}$ or $2 \mathrm{D}$ subspaces and bin them up to give images, energy spectra, or lightcurves. X-ray data files are usually photon-limited and sometimes the images, spectra, and lightcurves created from the event lists may have a few or even no photons in many bins.

Each of described above binned datasets requires its own calibration products. For example, an image analysis uses exposure maps, which accounts the mirror and detector sensitivity across the field-of-view (taking into account any changes in pointing direction), point spread function (PSF) which accounts the probability that a photon of given energy and position is registered in a given image pixel. Energy spectral analysis uses response matrices, which account the probability that a photon of given energy is registered in a given channel. And many others instrumental calibrations, including a systematic error that affects the data analysis accuracy. If we have the misfortune to have a very high signal-to-noise then this systematic error may dominate. We cannot add the systematic to the statistical uncertainties because the systematic uncertainties are usually correlated.

It is significant to note that the data analysis techniques developed in other wavebands may not transfer to X-ray astronomy (see, for example, recent papers by Konovalenko et al., 2016; Laporte et al., 2017; Mommert et al., 2016; Poole et al., 2008; Savanevych et al., 2015, 2018; as well as data analysis tool kits of the ground-based and space-born projects in various spectral ranges). Assuming both imaging and spectroscopic data, we are able to select the required "events" corresponding to a specific region of interested objects using a "region filter". From these events we are able to build spectra and fit them in a spectral package such as, for example, Xspec for X-ray data image analysis. Simple geometric shapes, such as annuli, boxes, sectors, ellipses, etc. can be used to assign a region filter. Assuming spherical symmetry and using annuli we can account for projection in galaxy clusters. However, we should note that the most extended sources are not symmetric.

In this paper we provide a brief overview of the Chandra X-ray data processing, including specific challenges of X-ray image and spectra processing. Here, we explore image and spectra processing techniques of three galaxy clusters A2029, A2107, A2151 observed by the Chandra to study their morphological diversity by different methods.

\section{Sample}

Our sample includes three galaxy clusters observed by Chandra X-ray Observatory. The list of objects and their coordinates, number of observations, exposure, redshift, etc. are presented in Tab. 1. We select X-ray observations with at least $10 \mathrm{ks}$ exposure time to investigate different methods of X-ray image analysis. The multiple observations for one object were combined. The information for these selected objects was taken from $\mathrm{NED}^{1}$, Simbad ${ }^{2}$, and HyperLEDA ${ }^{3}$ databases.

\footnotetext{
${ }^{1}$ https://ned.ipac.caltech.edu/

${ }^{2}$ http://simbad.u-strasbg.fr/

${ }^{3}$ Lyon-Meudon Extragalactic Database
} 


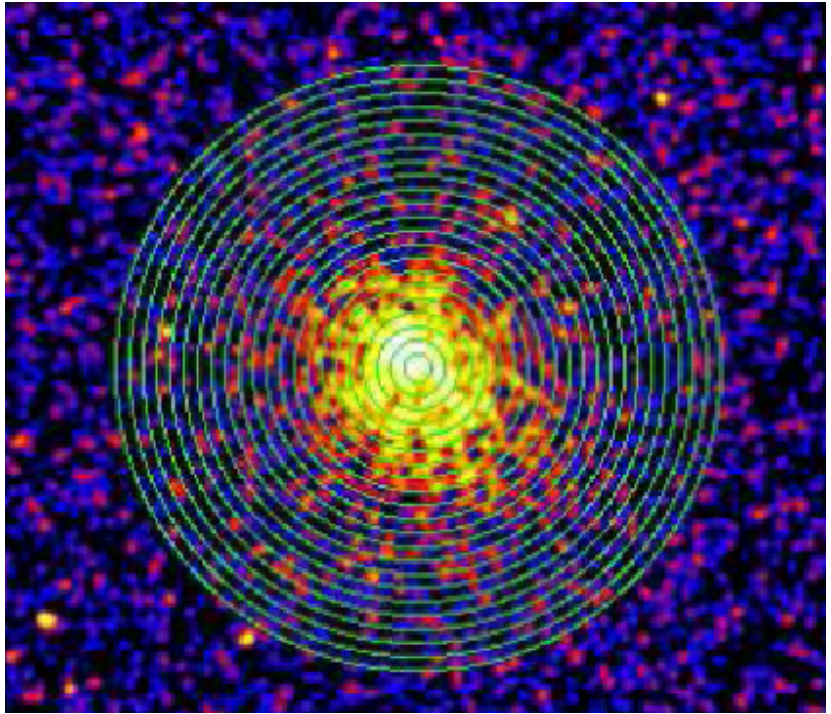

Рис. 1: The 0.5-7.0 keV X-ray image of galaxy cluster A2029 splited on concentric annular regions.

Angular and luminosity distances were calculated using redshifts and a $\Lambda \mathrm{CDM}$ cosmology with following parameters: $H_{0}=70 \mathrm{~km} / \mathrm{s} / \mathrm{Mpc}, \Omega_{m}=0.3$, and $\Omega_{\Lambda}$ $=0.7$. All the studied galaxy clusters were observed with ACIS instrument using chips 3 and 7.

\section{Data analysis}

The Chandra data were reduced following our previous analysis presented in papers by Babyk et al., 2012a,b,c,d,e; Babyk \& Vavilova, 2013, 2014a,b; and Babyk et al., 2014, 2018a,b,c; Pulatova et al., 2015; Vavilova et al., 2015. Here we summarize this analysis briefly: we used CIAO v.4.1-4.8 software packages with the latest calibration files; we applied chandra_repro tool to extract the level-2 cleaned event files; we also operated with wavdetect tool to identify and delete point sources; the background flares and time-dependent gains were removed as well.

\subsection{Radial profiles}

In this section we describe how we built the spatially resolved radial profiles of X-ray data for these galaxy clusters. Using the cleaned event files we constructed the X-ray images in the $0.5-7.0 \mathrm{keV}$ energy band. These images were splited on concentric annular regions how it is shown in Fig. 1. We note that there are two conventional methods to split images on concentric rings. In the first case, the X-ray image can be divided on the annuli with the same number of X-ray counts/photons in each annulus. In the second case, the X-ray images can be divided with increased number of X-ray photons in each annuli starting from central annulus. This latter case is used to decrease the accuracy of spectral parameters obtained from the X-ray spectra of each an- nular region. We note that both methods are generally accepted for an accurate spectral analysis.

The next step is the extraction of the X-ray spectra from each annuli. We used dmextract tool to get the Chandra spectra and we also apply this tool to obtain arf and rmf files. The spectra were extracted from annuli, avoiding the point sources and other asymmetric features. One of these spectra for galaxy cluster A2029 within 0.4-7.0 keV energy range is shown in Fig. 2 and was composed with a spectral software package Xspec v.11-12. This package consists of different models to describe thermal and non-thermal components of X-ray spectra. In the case of galaxy clusters, we have deals with thermal emission from the hot diffuse plasma with a temperature of millions Kelvin. Here we use phabs*apec model to fit each spectrum. The apec model fits the thermal emission from the hot atmosphere, while phabs models is component to account the photoelectric absorption and usually is fixed. Here we use column densities given in the last column of Tab. 1. These values were taken from Dickey \& Lockman (1990). The apec model includes a few parameters, both free and fixed. In our case we left the temperature and metalicity as the free parameters, and the column density and redshift as the fixed parameters. The fitting was verified using two statistics applied in Xspec, $\chi^{2}$ and Poisson statistics. Here we used $\chi^{2}$. The result of such a fitting as well as the quality of fit are given in Fig. 2. All the spectra were well fitted by the above described model with an average $\chi^{2}$ around 1 .

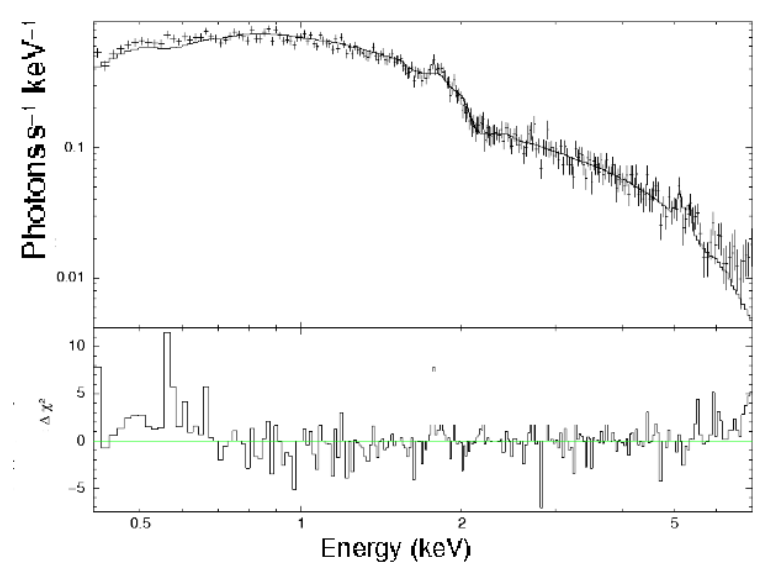

Рис. 2: The X-ray spectrum of annular region of galaxy cluster A2029 fitted by thermal model within 0.4-7.0 $\mathrm{keV}$ energy range.

The best-fit temperatures and metalicities of all the spectra are shown in Fig. 3 for all three galaxy clusters. We note that these profiles were obtained from the 2-dimensional images by projected spectra. These spectra are the model dependent, producing unphysical and large uncertainties of temperature, metalicity, density, and, as a result, gas and total mass profiles. 
Табл. 1: The list of galaxy clusters with their physical characteristics.

\begin{tabular}{ccccccc}
\hline Name & $\begin{array}{c}\alpha \\
\mathrm{J} 2000\end{array}$ & $\begin{array}{c}\delta \\
\mathrm{J} 2000\end{array}$ & ObsID & $\begin{array}{c}\text { Exposure } \\
\mathrm{ks}\end{array}$ & $z$ & $\begin{array}{c}N_{H} \\
10^{20} \mathrm{~cm}^{-2}\end{array}$ \\
\hline A2029 & 6.505 & 50.54 & 4977,6101 & $78.9,10.0$ & 0.077 & 3.07 \\
$\mathrm{~A} 2107$ & 34.39 & 51.48 & 4960 & 36.0 & 0.041 & 4.53 \\
$\mathrm{~A} 2151$ & 31.58 & 44.52 & 4996 & 22.1 & 0.036 & 3.49 \\
\hline
\end{tabular}

In this case, the deprojection, an approach to get "real" physical parameters from the $3 \mathrm{D}$-image, is required. There are a few different techniques to do this.

Here we apply a Direct Spectral Deprojection (DSDeproj) routine (see, Russell et al., 2008) to deproject our projected spectra. DSDeproj is a modelindependent method, which assumes only spherical symmetry. This approach works as follows. DSDeproj takes projected spectra obtained from a series of annuli and suitable backgrounds. Then, DSDeproj 1) subtracts the equivalent background spectrum from each spectra, 2) divides the count rate in each spectral energy bin by the volume, 3) scales the spectrum per unit volume from the outer annular region by the volume projected on to the neighbouring inner annular region, 4) subtracts this from the count rate in each spectral energy bin of that annular region, 5) calculates a new count rate per unit volume in each spectral energy bin for this annular region, and 6) subtracts off the projected of each outer annulus from the inner annuli to produce a number of deprojected spectra. Russell et al. (2008) used a Monte Carlo approach, repeating 6000 times, to calculate the uncertainties in the count rate of each spectral bin in each spectrum. They also assumed Gaussian errors due to the binning of input foreground cluster and background spectra with 200 counts per spectral bin.

The resulting spectra can then be modeled in $\mathrm{X}$ spec to produce the deprojected temperature and metalicity profiles. These profiles are presented in Fig. 3 as the red points. We found that our deprojected profiles are stable for any choice of radial binning. Any instability is visible, when a particular radial bin contains dramatically different hot gas properties, for example, cold fronts or shocks. The deprojected spectra produce smother profiles compared to those produced by projected spectra.

\subsection{Contour binning}

Local instabilities or variations in the temperature and metalicity profiles of galaxy cluster can be traced using the maps of their image created with different approaches. One of these approaches is called as a "contour binning" and is an algorithm for binning X-ray data using contours on an adaptively smoothed map.
The detailed description of contbin ${ }^{4}$ technique is presented by Sanders (2006). Here we summarize it briefly.

Physical characteristics (for example, temperature, metalicity, and density) usually vary in the direction of surface brightness varies. "Contour binning" routine uses the surface brightness to identify bins that cover areas of similar brightness. Such technique provides a great opportunity to measure easily the significance of individual spatial features. To apply this method we firstly need to get an estimate of the surface brightness in the image, avoiding of noise and counting statistics. Simple Gaussian smoothing cannot define the small brightness variations. Currently, there are a few methods to get an adaptively smoothed image using surface brightness, namely asmooth (Ebeling et al., 2006), csmooth (used in CIAO and based on asmooth), etc. To smooth the image with a top-hat kernel is a simple routine. The size of this kernel varies as a function of position and contains a minimum signal-to-noise ratio. It is also needed the color maps to apply the "contour binning" method. Color maps show the ratio of counts in two different bands. These maps are very useful in X-ray analysis, because the trends in X-ray color map follow physical parameter's trends such as temperature and metalicity. To generate the color maps the accumulative smoothing described above can also be used. All steps above as well as an adaptive smoothing routine is summarized in contbin. This algorithm works in the following way. The algorithm adds neighbouring pixels to a bin, starting at the highest flux pixel on a smoothed image. The algorithm stops to add those pixels when the signal-to-noise ratio exceeds an input threshold. As a result, a new bin is then created.

We need then to extract spectra from those bins and fit them to get the physical parameters. All the programming codes to perform such fitting can be found in the link presented above. In the case of our sample, we performed all the steps described above and built the galaxy cluster emission maps, which were grouped into bins that closely follow their surface brightness. The minimal signal-to-noise $(\mathrm{S} / \mathrm{N})$ ratio was selected of 60 for temperature maps to get maps with a high spatial resolution. This ratio corresponds to 3600 net

${ }^{4} \mathrm{C}++$
routine programming codes
xray.ast.cam.ac.uk/papers/contbin/



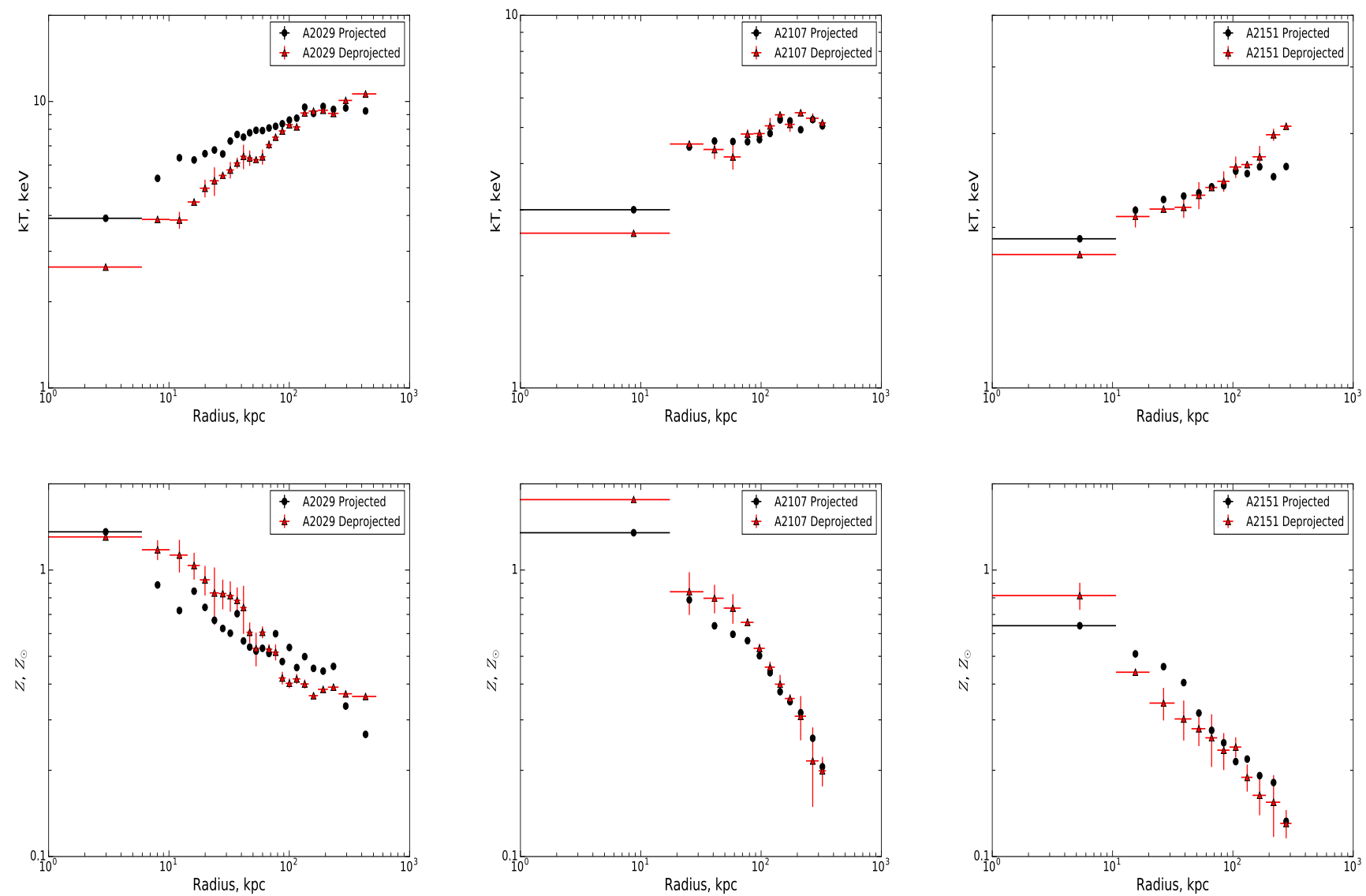

Рис. 3: The projected (black) and deprojected (red) temperature (top row) and metalicity (bottom row) profiles of galaxy clusters A2029, A2107, A2151.

counts. In case of the metalicity maps, we choose 80 (3600 net counts) as the $\mathrm{S} / \mathrm{N}$ ratio since accurate metalicity measurements require more counts. The X-ray spectra were extracted from each bin and were fit using the thermal model described above for the radial projected and deprojected spectra. The resulting maps (Fig. 4) are in agreement with radial profiles.

All the three temperature maps as well as the metal abundance maps clearly demonstrate how is important the count rate (statistics) and exposure time of X-ray observations to build such a type of map. The total exposure time of X-ray image of A2029 is about 90 $\mathrm{ks}$, while for other clusters they are about 30 and 20 ks. As a result, the temperature and metalicity maps of A2029 consist of bigger number of temperature and metal abundance sectors (see top-left and top-bottom plots in Fig. 4) as compared to those with less exposure time (central and right plots in the same figure). Bigger number of sectors helps to understand a spatial distribution of different thermodynamic properties. A2107 and A2151 galaxy clusters maps show narrow temperature and metalicity distributions. However, the temperature map of A2029 shows an evidence of shock at the cluster core. Looking into this map, we conclude that A2029 is a cooling flow cluster, showing clear drop of temperature into the cluster center. We see an obvious temperature jump between the preand post-shock bins along the full shock front. The central metal abundance of A2029 is about $1.0 Z_{\odot}$ and decreases with radius until $0.3-0.4 Z_{\odot}$. We assume that a clear distribution of metalicity in the direction south-west to north-east is seen towards the radio jet. The obtained maps are consistent with recent work of Hogan et al. (2017), where the similar distribution of metalicity was obtained for A2151 galaxy cluster. Hogan et al. (2017) argued that such distribution is a result of cavity moves, namely, the X-ray cavities may lift metals to larger radii during their rice inside the cluster.

\subsection{Wavelet analysis}

The two-dimensional radial wavelet analysis is very informative for detecting the substructures in the galaxy cluster structure. One of this method, the Mexican Hat as a basic platform for the programming code, was introduced by Slezak et al. (1990), Escalera \& Mazure 

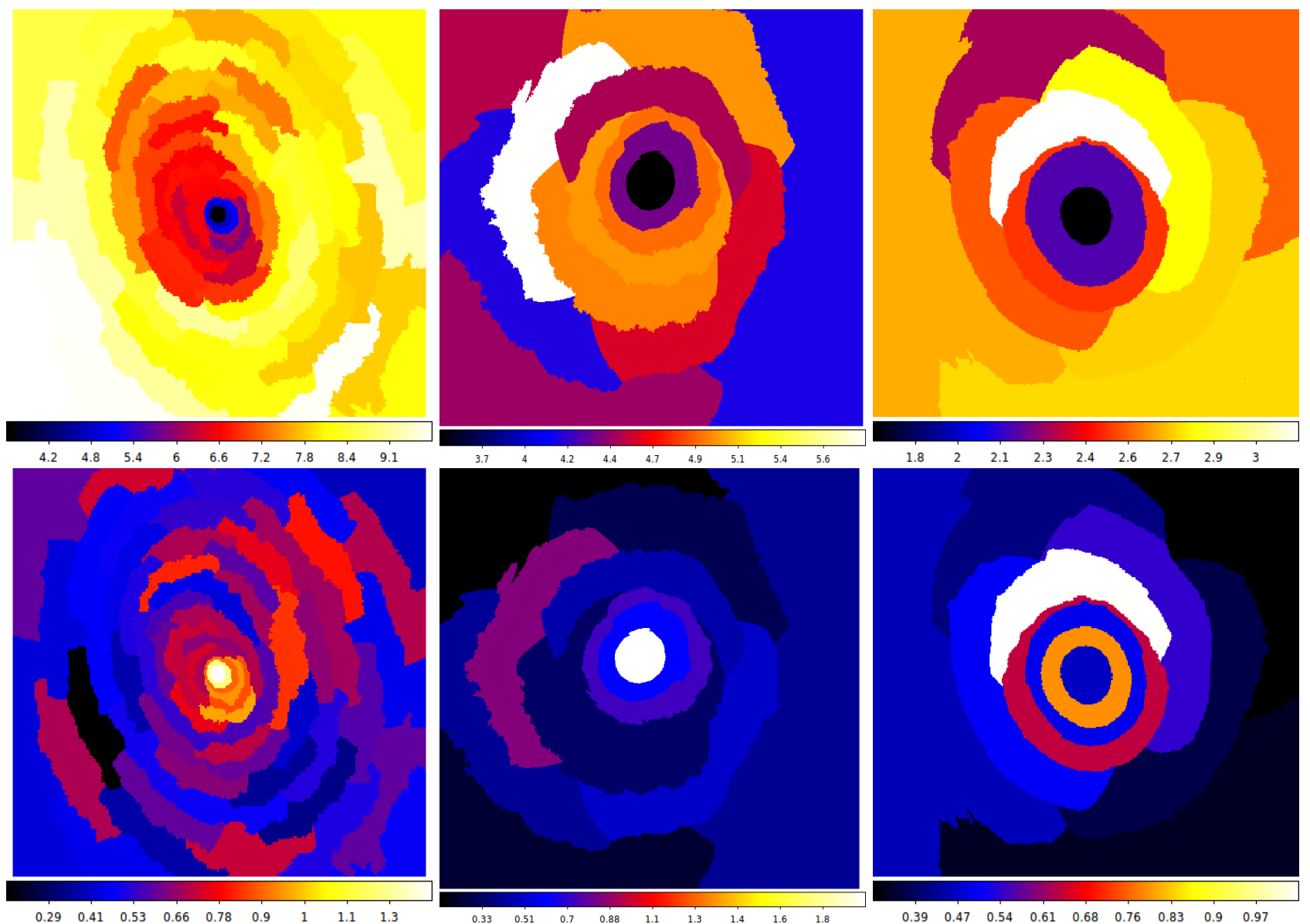

$\begin{array}{lllllllll}0.39 & 0.47 & 0.54 & 0.61 & 0.68 & 0.76 & 0.83 & 0.9 & 0.97\end{array}$

Рис. 4: The temperature (top) and metalicity (bottom) maps of A2029, A2107, and A2151 galaxy clusters (from left to right) obtained by contbin algorithm.

(1992) to detach galaxies in the cluster while analyzing its imagery. The dominant condition that the imagery field has covered the size of galaxy cluster (Escalera et al., 1994; Flin \% Vavilova, 1995, 1997; Vavilova \& Flin, 1997) was adopted for imageries in visible spectral bands but this is an exact case for X-ray images of clusters. The wavelet algorithms process the data at different scales. In case of substructure search, the task is to convolute the two-dimensional object distribution (signal function $s(r)$ ) on a grid $N x N$ pixels by the analyzing wavelet $F(r, a)$, where $a$ denotes a scale of the wavelet. This parameter determines the extent of spreading the object distribution in the space of wavelet coefficients $W C$ and allows to make a further analysis operating only with these coefficients. It is interesting that the Mexican Hat has a radial shape but it's able to detect non-circular substructures (Vavilova, 1997). Among the papers, where different platforms of wavelet analysis were used for detection of subclustering, mainly in the Abell clusters, or for analysis of turbulent processed in the ICM, we note as follows by Flin \& Vavilova, 1997; Gambera et al.; Bardelli et al., 1998; Shao Zheng-yi \& Zhao Jun-liang, 1999; Flin \& Kry- wult, 2006; Rostagni, 2012; Schwinn et al., 2018; Shi et al., 2018. The algorithm of substructure detection can be described briefly as follows:

1) determination of the center on the cluster's image;

2) normalization of the data through linear transformation into the range $[-1,1]$ yielding the radius of analyzed field $R_{f}=1$;

$3)$ search for scale of wavelet using the Mexican Hat formula: $F(r, a)=\left(2-r^{2} / a^{2}\right) \exp \left(-r^{2} / a^{2}\right)$, where $a$ is the wavelet's scale, $r$ is the distance between the center and point $(x, y)$, where the Mexican Hat is calculated;

4) analysis of superpositions of the Mexican Hats on the $N \times N$ pixel grid, which substituted the distribution of coordinates of galaxy cluster's components from which the X-ray radiation has been emitting;

5 ) the choice of wavelet scale $a$ is due to condition that the smallest scale $a=0.01 R_{f}$ can correspond to the individual galaxies or the regions of intracluster gas, while the scale $a=0.25 R_{f}$ could correspond to the central part of cluster, especially in case of the rich Abell clusters;

6) to verify results using, for example, the MonteCarlo simulations. 
So, we are able to detect the denser regions and the smallest substructures in cluster's components in relation to their coordinates using the Mexican Hat as the wavelet analysis approach. By other words, if the standard methods and contour binning methods allow us to construct profiles of the physical parameters with radii, then the wavelet analysis likely the Mexican Hat or a'true algorithm allow to select regions at the smallest scales in the distribution of these parameters and to get the coordinates of relevant components of galaxy cluster.

\section{Discussion and conclusions}

The X-ray Chandra Observatory is an unprecedented telescope with high-quality imaging and spectra capabilities that provides an extraordinary window into the high-energy Universe. Here we applied different approaches to three galaxy clusters A2029, A2107, and A2151 observed by Chandra to present the current possibilities of the X-ray data analysis. Our clusters have a component of cooler gas and/or complex of different substractures in their cores. We built a spatially resolved temperature and metalicity profiles for both cases (projected and deprojected). We applied DSDeproj routine to produce the deprojected spectra of our sample.

We also applied the "contour binning" method and showed that this method reliably creates the bins, which follow the surface brightness. We applied contbin algorithm to Chandra observations of these galaxy clusters. contbin algorithm is an ideal, where spectral changes are associated with changes in surface brightness, as is often the case in X-ray astronomy. Using contbin algorithm it has been found many important scientific results. For example, Fabian et al. (2005) defined a complex structure of the Centaurus cluster, Sanders et al. (2005) detected the non-thermal emission of a high metal shell associated with a radio babble in the Perseus cluster, Fabian et al. (2006) found an evidence of temperature changes associated with shock features, Vantyghem et al. (2014) found X-ray cavities associated with an elliptical shock front.

The application of various wavelets transforms is commonly used in astronomical tasks. Mostly the wavelet analysis is applied for search of quasiperiodicities or the characteristics timescales as compare with traditional Fourier-based methods. For example, Hovatta et al. (2008) confirmed the efficiency of the Morlet wavelet transform for analysis of flux curves of 80 active galactic nuclei (AGNs) in their time series at high radio frequencies, when different time scales in their activity were registered. We note that search for such quasi-periodicities is important in relation to constraints on the masses of black holes in galactic X-ray binaries. Another appropriate example of the informativeness of wavelets, namely the Continuous wavelet transform and the Cross wavelet transform, has been demonstrated by Espaillat et al. (2008) in their analysis of AGN X-ray time variability data obtained with the XMM-Newton and detection of a quasi-period for $3 \mathrm{C} 273$. Because of this period affected the estimation of the black hole mass (the estimate was lower than that by reverbation-mapping methods), the authors concluded that X-ray quasi periodicities would be caused by a higher order oscillatory mode of the accretion disk. Such approach could be useful while the multi-wavelength properties from gamma to radio spectral bands have to be considering in frame of AGN's long-year monitoring programs (see, Breedt et al., 2010; Volvach et al., 2011; Chesnok et al., 2009; Ryabov et al., 2016; and others works) or for analysis of web structure of the Universe (Einasto et al., 2011; ArnalteMur et al., 2012).

Since the end of 1990ies the application of wavelet transforms to the detachment of substructures in the X-ray galaxy clusters has activated with the use of the ROSAT and other space-born X-ray observatories data (see, for, example, early works by Slezak et al., 1994; Starck \& Pierre, 1998) and has followed till now (see, for example, Finoguenov et al.,, 2007; Bozkurt, 2009; Akamatsu et al., 2016). Such approach allows to consider also the cores of cluster (mostly virialized) as deeply as to the presence of $\mathrm{cD}$ galaxy as well as to find small-scale inhomogeneities related to the intracluster media and cooling flows. In our opinion, the detachment of subclustering in X-ray galaxy clusters should precede further research because of its existence imposes doubt in the validity of the hydrostatic equilibrium hypothesis of the X-ray gas when the dynamical matter distribution and the profiles of various physical parameters along radii are considered. In respect to this, we described the algorithm of applying the wavelet analysis based on the Mexican Hat programming code, which is successfully used to distinguish substructures in the distribution of X-ray emission parameters while analyzing the X-ray imagery of galaxy clusters. Its application altogether with the standard methods of processing the X-ray image and contour binning methods can significantly complement our understanding of the physical processes that occur in X-ray galaxy clusters.

Acknowledgements This research has made use of the data obtained from the Chandra Data Archive and the Chandra Source Catalog, and software provided by the Chandra X-ray Center (CXC) in the application packages CIAO, ChIPS, and Sherpa. We thank all the staff members involved in the Chandra project. Additionally we have used NED, Simbad, HyperLEDA, and ADS facilities. This work was partially supported in frame of the Target Complex Program of the NAS of Ukraine for Space Scientific Research (2018-2022). 


\section{References}

Arnalte-Mur P., Labatie A., Clerc N. et al.: 2012, A\& A, 542, id.A34, 11 pp.

Arnaud K. A.: 1996, ADASS V, ASP Confer. Ser., 101, 17

Akamatsu H., Gu L., Shimwell T.W. et al.: 2016, $A \mathscr{E} A$, 593, id.L7, 5 pp.

Babyk Iu. V., Vavilova I. B.: 2012a, Odessa Astron. Publ., 25, 119

Babyk Iu., Melnyk O., Elyiv A.: 2012b, AASP, 2, 56

Babyk Iu. V.: 2012c, Bull. CrAO, 108, 87

Babyk Iu. V., Elyiv A., Melnyk O., Krivodubskij V.

N.: 2012d, Kinemat. Physics Celest. Bodies, 28, 69

Babyk Iu.V.: 2012e, J. Physical Studies, 16, 1904

Babyk Iu.V., Vavilova I.B.: 2013, Odessa Astron. Publ., 26, 175

Babyk Yu V., Del Popolo A., Vavilova I.B.: 2014, Astron. Reports, 58, 587

Babyk Iu.V., Vavilova I. B.: 2014a, Astrophys. \& Space Sci., 349, 415

Babyk Iu.V., Vavilova I.B.: 2014b, Astrophys. \& Space Sci., 353, 613

Babyk Iu.V., McNamara B.R., Nulsen P.E.J. et al.: 2018a, ApJ, 857, 32

Babyk Iu.V., McNamara B.R., Nulsen P.E.J. et al.: 2018b, ApJ, 862, 39

Babyk Iu.V., McNamara B.R., Tamhane P.D. et al.: 2018c, submitted to ApJ, arXiv:1810:11465

Bardelli S., Pisani A., Ramella M. et al.: 1998, MN$R A S$, 300, 2, 589

Bozkurt M., Hudaverdi M., Ercan E.N.: 2009, Publ. Astron. Obs. Belgrade, 86, 301

Breedt E., McHardy I.M., Arvalo P. et al.: 2010, MN$R A S, 403,2,605$

Chesnok N.G., Sergeev S.G., Vavilova I.B.: 2009,

Kinemat. Physics Celest. Bodies, 25, 2, 107

Dickey J.M., Lockman F.J.: 1990, ARAA, 28, 215

Ebeling H., White D.A., Rangarajan F.V.N.: 2006, MNRAS, 368, 65

Einasto J., HГjtsi G., Saar E. et al.: 2011, $A \mathscr{E} A$, 531, id.A75, 11 pp.

Escalera E., Mazure A.: 1992, ApJ, 288, 23

Escalera E. et al.: 1994, ApJ, 423, 539

Espaillat C. et al.: 2008, ApJ, 679, 182

Fabian A.C., Sanders J.S., Taylor G.B., Allen S.W.: 2005, MNRAS, 360, 20

Fabian A.C., Sanders J.S., Taylor G.B. et al.: 2006, MNRAS, 366, 417

Finoguenov A., Guzzo L., Hasinger G. et al.: 2007, ApJ SS, 172, 1, 182

Flin P, Vavilova I.B.: 1995, SISSA Ref. 65/95/A,

World Scientific Press, p. 117-122

Flin P., Vavilova I.B.: 1997, Astrophys. Letters 8 Communications, 36, 113

Flin P., Krywult J.: 2006, A\&A, 450, 1, 9

Gambera M., Pagliaro A., Antonuccio-Delogu V., Becciani, U.: 1997, ApJ, 488, 1, 136
Hogan M.T., McNamara B. R., Pulido F. A. et al.: 2017, ApJ, 851, 66

Hovatta T. et al.: 2008, $A \& A$, 488, 3, 897

Laporte N., Bauer F.E., Troncoso-Iribarren P. et al.: 2017, A\& A, 604, id.A132, 16 pp.

Konovalenko A., Sodin L., Zakharenko V. et al.: 2016, Experimental Astronomy, 42, 1, 11

Mommert M., Moskovitz N., Trilling D.E.: 2016, AAS DPS meeting, 48, id.123.42

Poole T.S., Breeveld A.A., Page M.J. et al: 2008, $M N R A S, \mathbf{3 8 3}, 2,627$

Pulatova N.G., Vavilova I.B., Sawangwit U. et al.: 2015, MNRAS, 447, 2209

Pulido F.A., McNamara B.R., Edge A.C. et al.: 2018, ApJ, 853, 177

Rostagni F., Benoist C., Maurogordato S.: 2012, SF2A-2012, 477

Russell H.R, Sanders J.S., Fabian A.C.: 2008, MN$R A S$, 390, 1207

Ryabov M.I., Sukharev A.L., Donskykh H.I.: 2016, Radio Physics \& Radio Astronomy, 21, 161

Sanders J.S., Fabian A.C., Dunn R.J.H.: 2005, $M N$ $R A S, \mathbf{3 6 0}, 133$

Sanders J.S.: 2006, MNRAS, 371, 829

Sanders J.S., Fabian A.C., Allen S.W.: 2000, MN$R A S, \mathbf{3 1 8}, 733$

Savanevych V.E., Briukhovetskyi O.B., Sokovikova N.S. et al.: 2015, MNRAS, 451, 3, 3287

Savanevych V.E., Khlamov S.V., Vavilova I.B. et al.: 2018. $A \& A$, 609, id.A54, 11 pp.

Schwinn J., Baugh C. M., Jauzac M. et al.: 2018, MNRAS, bf 481, 4, 4300

Shao Zheng-yi, Zhao Jun-liang: 1999, Chinese Astron. Astrophys., 23, 2, 159

Shi Xun, Nagai D., Lau E.T.: 2018, MNRAS. 481, 1, 1075

Slezak E., Bijaoui M., Mars G.: 1990, A\&A, 227, 301

Slezak E., Durret F., Gerbal D.: 1994, AJ, 108, 6, 1996

Starck J.-L., Pierre M.: 1998, ADASS VII, ASP Confer. Ser., 145, 501

Tashiro M., Maejima H., Toda K. et al.: 2018b, Proc. SPIE, 10699, id.1069922

Vantyghem A.N., McNamara B.R., Russell H.R. et al.: 2014, MNRAS, 442, 3192

Vavilova I.B., Flin P.: 1997, In ADASS VI, ASP Confer. Ser., 125, 186

Vavilova I.B.: 1997, Data Analysis in Astronomy, Proc. 5th Workshop, World Scientific Press, 297

Vavilova I.B.: 2000, Kinemat. Fiz. Nebesn. Tel, Suppl. no. 3, 155

Vavilova I. B., Vasylenko A.A., Babyk Iu.V., Pulatova N. G.: 2015, Odessa Astron. Publ., 28, 150

Vol'Vach A.E., Vol'Vach L.N., Kut'kin A.M. et al.: 2011, Astron. Reports, 55, 7, 608 